(n)

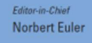
Journal of Nonlinear Mathematical Physics

\title{
On a spectral analysis of scattering data for the Camassa-Holm equation
}

Chueh-Hsin Chang, Tony Wen-Hann Sheu

To cite this article: Chueh-Hsin Chang, Tony Wen-Hann Sheu (2015) On a spectral analysis of scattering data for the Camassa-Holm equation, Journal of Nonlinear Mathematical Physics 22:1, 102-116, DOI:

https://doi.org/10.1080/14029251.2015.996443

To link to this article: https://doi.org/10.1080/14029251.2015.996443

Published online: 04 January 2021 


\title{
On a spectral analysis of scattering data for the Camassa-Holm equation
}

\author{
Chueh-Hsin Chang \\ Center of Advanced Study in Theoretical Sciences, \\ National Taiwan University, \\ Taipei, 10617, Taiwan \\ changjuexin@tims.ntu.edu.tw \\ Tony Wen-Hann Sheu * \\ Center of Advanced Study in Theoretical Sciences \\ Department of Engineering Science and Ocean Engineering \\ Institute of Applied Mathematical Science \\ National Taiwan University, \\ Taipei, 10617, Taiwan \\ twhsheu@ntu.edu.tw
}

Received 30 August 2014

Accepted 6 October 2014

\begin{abstract}
Physical details of the Camassa-Holm $(\mathrm{CH})$ equation that are difficult to obtain in space-time simulation can be explored by solving the Lax pair equations within the direct and inverse scattering analysis context. In this spectral analysis of the completely integrable $\mathrm{CH}$ equation we focus solely on the direct scattering analysis of the initial condition defined in the physical space coordinate through the time-independent Lax equation. Both of the continuous and discrete spectrum cases for the initial condition under current investigation are analytically derived. The scattering data derived from the direct scattering transform for non-reflectionless case are also discussed in detail in spectral domain from the physical viewpoint.
\end{abstract}

Keywords: direct and inverse scattering; Camassa-Holm equation; isospectral problem.

2000 Mathematics Subject Classification: 35Q35, 35Q40, 37K15, 37K10

\section{Introduction}

The Camassa-Holm $(\mathrm{CH})$ Equation

$$
u_{t}+2 \kappa u_{x}-u_{x x t}+3 u u_{x}=2 u_{x} u_{x x}+u u_{x x x}, x \in \mathbb{R}, t>0 .
$$

was derived in 1993 for modelling unidirectional wave propagation within the context of shallow water [5]. The field variable $u(x, t)$ in $\mathrm{CH}$ equation denotes the height of free surface above a flat bottom. The free parameter $\kappa>0$ has been known to be related to the critical shallow water speed. Eq. (1.1) was also derived by Fuchssteiner and Fokas [18] by the method using recursion operators. Johnson [22], Constantin et al. [13] and Ionescu-Krus [21] derived CH equation as models for shallow water by ways which are different from [5]. The $\mathrm{CH}$ equation subject to an initial condition

${ }^{*}$ Corresponding author 
given below will be considered

$$
u(x, 0)=u_{0}(x), x \in \mathbb{R} .
$$

The initial condition $u_{0}(x)$ and its first derivative $u_{0}^{\prime}(x)$ under current investigation are both assumed to decay sufficiently fast to zero, or $u_{0}(x) \rightarrow 0$ and $u_{0}^{\prime}(x) \rightarrow 0$, as $|x| \rightarrow \infty$ or at a point far from the origin of $x$.

One mathematical importance of the $\mathrm{CH}$ equation for $\kappa=0$ is that the peaked soliton solution $u(x, t)=c e^{-|x-c t|}$, which has been called as a peakon solution, is permitted for all $c \in \mathbb{R}$ (see [5]). Moreover, Camassa et al. [6] reported that smooth solitary wave solutions to (1.1) are permitted for all $\kappa>0$. There have been many analytical studies of (1.1-1.2) on the subjects, for example, about the existence of global solution [10], [8], weak solution [11], breaking wave solution [12], [8], stability of peaked soliton solution for $\kappa=0$ [14], and the solitary waves for $\kappa>0$ [15]. This equation is known as one of an important integrable equations because it has many features similar to the $\mathrm{KdV}$ equation. For example, the solvability of the initial value problem for the $\mathrm{CH}$ equation can be obtained by the direct and inverse scattering methods which were adopted earlier to study the KdV equation in the important work by Gardner et al. [19].

With the long time asymptotic behaviors obtained in [2], it is intuitive to see that if a wave function of the isospectral problem completely transmits, then its solution does not have oscillating tails. In other words, such a wave is simply propagated as a pure soliton. In this light, study of the corresponding scattering problem may help to understand more about the solution nature of $\mathrm{CH}$ equation since it is often theoretically impossible to get in the space-time domain. The scattering problem was analyzed under the condition permitting a continuous spectrum by Constantin [9]. On the contrary, Constantin and Strauss [15], Johnson [23], Lenells [24], Li and Zhang [25] studied only the reflectionless case permitting only a discrete spectrum. For this case the solution of $\mathrm{CH}$ equation can be well-constructed as $N$ soliton solutions. They supposed that the scattering data are known. However, the nonzero reflection coefficient case is still an open problem to the best of the authors' knowledge. From these previous works, we can ask the following questions:

(1) How to find an initial condition with non-zero reflection coefficient ?

(2) How the initial condition of $\mathrm{CH}$ equation influences the scattering data?

In this paper, we attempt to answer the above two questions by considering the isospectral problem from the Lax pair equations of $\mathrm{CH}$ equation. A specified initial condition will be shown in Section 2. This initial condition includes a given parameter $q_{0} \in(0,1)$ with a pulse-like profile decaying to zero as $|x| \rightarrow \infty$ (see (2.14)). Then the direct scattering problem is solved subject to the initial condition in (2.14). Through the Liouville transform, we can get a Schrödinger operator with a Delta function-like potential for the particularly chosen initial condition stated above. The scattering data corresponding to this potential can then be obtained explicitly with the nonzero reflection coefficient (see [29]). Such an initial condition with nonzero reflection coefficient is not found previously. Due to the difficulty of analytically integrating the nonzero reflection coefficient, the exact time-evolving solution of this initial value problem is normally unavailable. However, many interesting and useful information can be extracted from the direct scattering process. Subsequent to this work, we will compare the solution solved directly from the $\mathrm{CH}$ equation in space-time domain using the upwinding compact difference scheme and the asymptotics obtained from [2] and [1]. By varying the parameter $q_{0}$, the variation of the long-time asymptotics of solutions of $\mathrm{CH}$ equation will be illustrated. Although this is a special case to be studied, it is interesting to investigate the 
influence of the initial condition on the solution nature of integrable systems. In Section 2 we give the specified initial data with the corresponding scattering data. All of them depend on the parameter $q_{0} \in(0,1)$. In Section 3, we derive in detail the analytical scattering data. In Section 4, some conclusions drawn from this study and other works under investigation are summarized.

\section{Direct scattering analysis}

$\mathrm{CH}$ equation is equivalent to the compatibility condition between the following two Lax pair equations [5]:

$$
\begin{gathered}
\frac{1}{w}\left(-\psi_{x x}+\frac{1}{4} \psi\right)=\lambda \psi, \\
\psi_{t}=-\left(\frac{1}{2 \lambda}+u\right) \psi_{x}+\frac{1}{2} u_{x} \psi .
\end{gathered}
$$

In the above, the momentum variable $w$ is expressed as

$$
w=u-u_{x x}+\kappa .
$$

In this section some important points of the direct scattering theories are given. The detail can be seen, for example, in Faddeev's paper [17]. See also [26] and [2]. At $t=0$, we assume $w(x, 0)>0$. It was proved by Constantin [9] that under this constraint, $w(x, t)>0$ for $x \in \mathbb{R}$, for all $t>0$. We also require the boundedness of the following integral

$$
w(x, 0) \in\left\{v \in H^{3}(\mathbb{R}) \mid \int_{\mathbb{R}}(1+|x|)^{1+l}\left(|v(x)-\kappa|+\left|v^{\prime}(x)\right|+\left|v^{\prime \prime}(x)\right|\right) d x<\infty\right\},
$$

where $l \in \mathbb{N}$. While solving (2.1) for $t \geq 0$, we aim to eliminate $w$ from the left hand side of (2.1). The following Liouville transform [9] is therefore performed in the current analysis of the $\mathrm{CH}$ equation

$$
\begin{gathered}
\tilde{\psi}(y)=\left(\frac{w(x, t)}{\kappa}\right)^{\frac{1}{4}} \psi(x), \\
y=x-\int_{x}^{\infty}\left(\sqrt{\frac{w(r, t)}{\kappa}}-1\right) d r .
\end{gathered}
$$

The above transformation holds under the assumption (2.4), $\kappa>0$ and $w(x, t)>0$ mentioned above. Transformation of the Eqs. (2.5) and (2.6) yields

$$
L \tilde{\psi}:=-\tilde{\psi}_{y y}+q(y, t) \tilde{\psi}=k^{2} \tilde{\psi}
$$

where $\lambda=\frac{1}{\kappa}\left(\frac{1}{4}+k^{2}\right)$ and

$$
q(y, t)=\frac{w_{y y}(y, t)}{4 w(y, t)}-\frac{3}{16} \frac{\left(w_{y}\right)^{2}(y, t)}{w^{2}(y, t)}+\frac{\kappa-w(y, t)}{4 w(y, t)} .
$$

Note that $w(y, t)=w(x(y, t), t)$ with $x(y, t)$ being obtained from (2.6). 
Due to the prescribed condition in (2.4), from (2.8) the Faddeev's condition given below should be satisfied so as to ensure boundedness of the integral

$$
q(y, 0) \in\left\{q(y, 0) \in H^{1}(\mathbb{R})\left|\int_{\mathbb{R}}(1+|y|)^{1+l}\right| q(y, 0) \mid d y<\infty\right\} .
$$

We can now think in a way that there are discrete $\left(k^{2}<0\right)$ and continuous $\left(k^{2}>0\right)$ spectra of $L$. Under the Faddeev's condition (2.9), from [16, 17,26], for example, there exists a finite set of discrete simple eigenvalues $-\mu_{1}^{2}, \ldots,-\mu_{N}^{2}$ of $L$ for some $N \in \mathbb{N}$. For each eigenvalue $-\mu_{j}^{2}$, let $\psi_{j}(y)$ be the corresponding eigenfunction with $j=1, \ldots, N$. The normalization constant $\gamma_{j}$ is defined as

$$
\psi_{j}(y)=\gamma_{j} e^{-\mu_{j} y}+o(1) \text { as } y \rightarrow \infty
$$

with $j=1, \ldots, N$. The value of $\gamma_{j}$ is fixed by normalizing the eigenfunction according to:

$$
\int_{\mathbb{R}} \psi_{j}^{2}(y) d y=1
$$

For the continuous spectrum case, by virtue of the Faddeev's condition (2.9) and the classical scattering theory in [16] and [17], the asymptotic behavior of the wave function $\hat{\psi}\left(y ; k^{2}\right)$ (i.e., the eigenfunction) at a location far away from the origin can be described below for a right-running incident wave $e^{-i k y}$

$$
\begin{gathered}
\hat{\psi} \sim e^{-i k y}+\tilde{R}(k) e^{i k y} ; \text { as } y \rightarrow \infty, \\
\hat{\psi} \sim \tilde{T}(k) e^{-i k y} ; \text { as } y \rightarrow-\infty .
\end{gathered}
$$

The above set of equations describes the scattering of a solution from the right incident wave on the potential $q(y)$. In Eq. (2.11), $\tilde{R}(k)$ represents the reflection coefficient while $\tilde{T}(k)$ in Eq. (2.12) is denoted as the transmission coefficient.

By the Liouville transformation given in (2.5) and (2.6), one can similarly define the reflection coefficient $R(k)$ and the transmission coefficient $T(k)$ for the eigenfunction corresponding to the continuous spectrum of $H(t):=\frac{1}{w}\left(-\frac{d}{d x^{2}}+\frac{1}{4}\right)$. From [2], we can find the relation between $\tilde{R}(k)$, $\tilde{T}(k)$ and $R(k), T(k)$ as

$$
T(k)=\tilde{T}(k) e^{i k H_{-1}(w)}, R(k)=\tilde{R}(k) .
$$

where $H_{-1}(w)=\int_{\mathbb{R}}\left(\sqrt{\frac{w(r, t)}{\kappa}}-1\right) d r$. The time evolution of the data $R(k, t), \mu_{j}(t), \gamma_{j}(t)$ can be derived by (2.2) as the standard analysis carried out in many references such as $[2,16,17,26]$. By the isospectral property we have $\mu_{j}(t)=\mu_{j} .\left\{R(k, t), \mu_{j}, \gamma_{j}(t)\right\}$, where $j=1, \ldots, N$ for some $N \in \mathbb{N}$, is physically known as the scattering data of the operator $H(t)$.

In the following we consider a specified initial data $u(x, 0)$ of the $\mathrm{CH}$ equation. Let $q_{0} \in(0,1)$ be a given constant and $A=\frac{q_{0}}{1-q_{0}}$. Consider the $\mathrm{CH}$ equation (1.1) subject to the following initial 
condition

$$
u(x, 0)= \begin{cases}\frac{\kappa A\left(A+1+\ln \left(e^{x}-A\right)\right)}{e^{x}}, & \text { for } x \geq \ln (1+A), \\ \frac{\kappa A\left(A+1+\ln \left((1+A)^{2} e^{-x}-A\right)\right)}{(1+A)^{2} e^{-x}}, & \text { for } x<\ln (1+A) .\end{cases}
$$

Substitution of (2.14) into the definition of $w$ yields

$$
w(x, 0)= \begin{cases}\kappa\left(\frac{1}{1-A e^{-x}}\right)^{2}, & \text { for } x \geq \ln (1+A), \\ \kappa\left(\frac{(1+A)^{2}}{(1+A)^{2}-A e^{x}}\right)^{2}, & \text { for } x<\ln (1+A) .\end{cases}
$$

Moreover, two conditions can be obtained in the course of changing variables. From (2.6), we can express $y$ in terms of $x$ as follows

$$
y= \begin{cases}\ln \left(e^{x}-A\right), & \text { for } x \geq \ln (1+A), \\ -\ln \left((1+A)^{2} e^{-x}-A\right), & \text { for } x<\ln (1+A) .\end{cases}
$$

or

$$
x=x(y)= \begin{cases}\ln \left(A+e^{y}\right), & \text { for } y \geq 0, \\ -\ln \left(\frac{A+e^{-y}}{(1+A)^{2}}\right), & \text { for } y<0 .\end{cases}
$$

Substitution of the above equation for the change of variables into (2.15) yields $\left.w(x, 0)\right|_{x=x(y)}=$ $\kappa\left(1+A e^{-|y|}\right)^{2}$. We can then further get the following equation

$$
\frac{w_{y y}(y, 0)}{4 w(y, 0)}-\frac{3}{16} \frac{\left(w_{y}\right)^{2}(y, 0)}{w^{2}(y, 0)}+\frac{\kappa-w(y, 0)}{4 w(y, 0)}=-q_{0} \delta(y)=q(y, 0)
$$

where the last equality of the above equation is due to (2.8). Based on the transformation of the eigenvalue problem from (2.1) to (2.7), for the initial condition specified by (2.14) we are led to know that the eigenvalue problem (2.7) becomes

$$
-\tilde{\psi}_{y y}-q_{0} \delta(y) \tilde{\psi}=k^{2} \tilde{\psi} .
$$

From the reference [16], the scattering data of (2.18) can be obtained as

$$
R(k)=\frac{-q_{0}}{q_{0}+2 i k}, \mu=\frac{q_{0}}{2}, \gamma=\sqrt{\frac{q_{0}}{2}} .
$$

Before conducting the spectral analysis of the direct spectral problem, it is worthy to mention again that the $\mathrm{CH}$ equation cast in space-time domain has been transformed to the equation in wavenumber-frequency spectral domain. For the direct spectral problem, time has been fixed to be zero. The corresponding spectral analysis, as a result, involves only the wavenumber $k$ shown in Eq. (2.18).

Depending on the potential $q_{0} \delta(y)$, there are two types of linear Schrödinger equation for (2.18). They are characterized by their own spectral sets $\left\{k^{2}\right\}$. In the following spectral analysis, the values of $q_{0}$ under investigation are fixed at $\frac{1}{3}, \frac{1}{2}$ and $\frac{2}{3}$. Under the circumstances, the spectral 
solutions in association with the investigated potentials, which are $-\frac{1}{3} \delta(y),-\frac{1}{2} \delta(y)$ and $-\frac{2}{3} \delta(y)$, are solved in this study.

According to the expression of the momentum variable given in (2.15), we can get

$$
H_{-1}(w)=\ln (1+A)^{2}=-2 \ln \left(1-q_{0}\right) .
$$

Having derived $H_{-1}(w)$, as $x \rightarrow-\infty$ the equation given by $y \sim x-H_{-1}(w)=x-\ln (1+A)^{2}$ can be derived thanks to (2.6). It is therefore implied that $x$ and $y$ have the same slope at $x \rightarrow-\infty$. This can be seen from the three lines having the constant slope schematically shown in Figure 1. For the cases $q_{0}=\frac{1}{3}, \frac{1}{2}$, (i.e., $A=1$ ) and $\frac{2}{3}$, the corresponding changes of $y$ with respect to $x$ at $\kappa=1$ are plotted in Figure 1. Indeed, by writing (2.16) as $y=y\left(x ; q_{0}\right)$, we can find that

$$
\frac{\partial y\left(0 ; q_{0}\right)}{\partial q_{0}}=\frac{-(2 A+1)}{\left(A^{2}+A+1\right)\left(1-q_{0}\right)^{2}}<0 .
$$

Hence the $y$-intercept is increased as well, as shown in Figure 1, as $q_{0}$ increases.

By substituting the expression (2.17) into (2.14), we can rewrite the initial condition for $u$ in terms of $y$ as

$$
u(y, 0)=\frac{\kappa A(|y|+A+1)}{\left(A+e^{|y|}\right)} .
$$

Moreover, as shown in Figure 2 (a) and 2 (c), both $w(y, 0)$ and $u(y, 0)$ are known to be symmetric with respect to $y=0$. In the $x$ coordinate, as shown in Figure 2 (b) and $2(\mathrm{~d})$, both $w(x, 0)$ and $u(x, 0)$ are symmetric about the line $x=\ln (1+A)$. It can be observed from Figure 2 (a) that $w(y, 0)$ has a peak-like profile. In the $x$ coordinate, $w(x, 0)$ plotted in Figure 2 (b) has a peak-like profile as well. By writing $w(y, 0)=w\left(y, 0 ; q_{0}\right)$, we can find that

$$
\max _{y \in \mathbb{R}} w\left(y, 0 ; q_{0}\right)=w\left(0,0 ; q_{0}\right)=\kappa\left(\frac{1}{1-q_{0}}\right)^{2}, \frac{\partial w\left(0,0 ; q_{0}\right)}{\partial q_{0}}=\frac{2 \kappa(1+A)}{\left(1-q_{0}\right)^{2}}>0 .
$$

As a result, $\max _{y \in \mathbb{R}} w\left(y, 0 ; q_{0}\right)$ is increasing w.r.t. $q_{0}$. Moreover, as $q_{0} \rightarrow 0, \max _{y \in \mathbb{R}} w\left(y, 0 ; q_{0}\right) \rightarrow \kappa$; while as $q_{0} \rightarrow 1, \max _{y \in \mathbb{R}} w\left(y, 0 ; q_{0}\right) \rightarrow \infty$. For the $w$ expressed in $x$ domain, the result is similar. For $u(y, 0)=u\left(y, 0 ; q_{0}\right)$ in $(2.20)$, we can also find that

$$
\max _{y \in \mathbb{R}} u\left(y, 0 ; q_{0}\right)=u\left(0,0 ; q_{0}\right)=\kappa \frac{q_{0}}{1-q_{0}}, \frac{\partial u\left(0,0 ; q_{0}\right)}{\partial q_{0}}=\frac{\kappa}{\left(1-q_{0}\right)^{2}}>0
$$

Hence as $q_{0} \rightarrow 0, \max _{y \in \mathbb{R}} u\left(y, 0 ; q_{0}\right) \rightarrow 0$; while as $q_{0} \rightarrow 1, \max _{y \in \mathbb{R}} u\left(y, 0 ; q_{0}\right) \rightarrow \infty$.

\section{Exploration of scattering wave details}

Define the Hamiltonian operator as $\hat{H}=-\partial_{y y}^{2}+q(y)$, one can rewrite Eq. (2.7) as $\hat{H} \tilde{\psi}=k^{2} \tilde{\psi}$, implying that an application of the operator $\hat{H}$ on the wavefunction $\tilde{\psi}$ yields a term proportional to the same wavefunction. As a result, $\tilde{\psi}$ is a stationary state and the proportionality, $k^{2}$, denotes the energy of the state $\tilde{\psi}$. More precisely, the solution $\tilde{\psi}$ of (2.7) is called as the energy eigenstate with the corresponding energy $k^{2}$ [27]. Eq. (2.7) is regarded as the eigenvalue equation for the Hamiltonian operator $\hat{H}$. This equation for the wavefunction $\tilde{\psi}$ minimizes the total energy of a many-electron system in an electric field. 


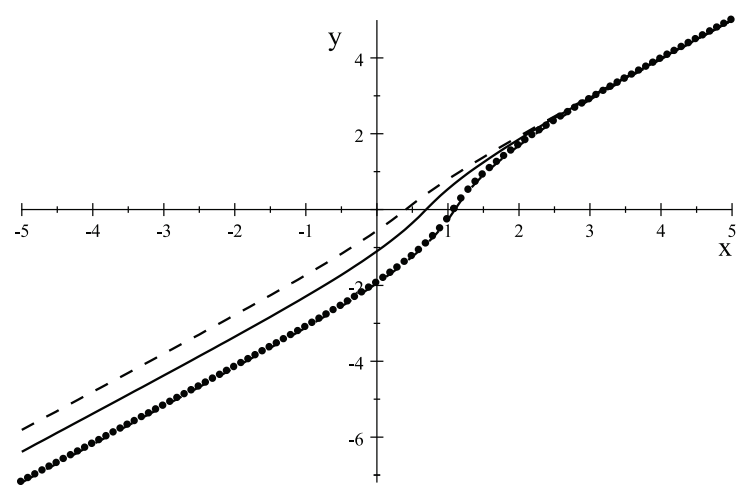

Fig. 1. The plot of coordinate $y$ defined in (2.6) with respect to the coordinate $x$ at $q_{0}=\frac{1}{3}$ (dash line), $\frac{1}{2}$ (solid line), $\frac{2}{3}$ (dot dash line) and $\kappa=1$.

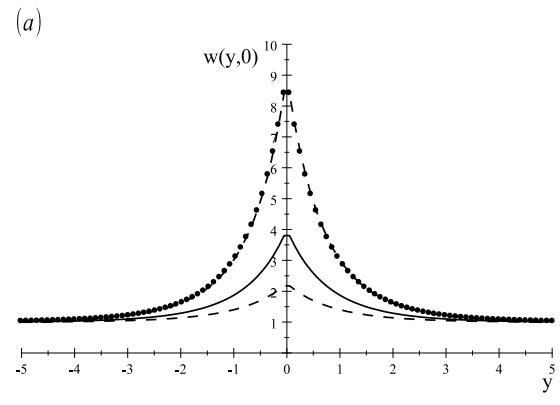

(c)

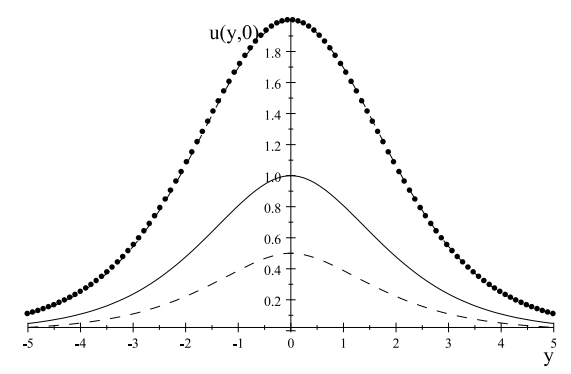

$(b)$

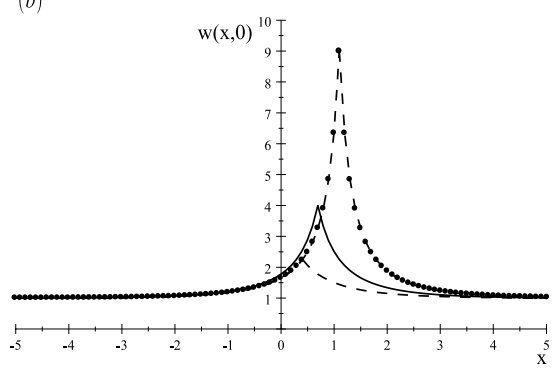

$(d)$

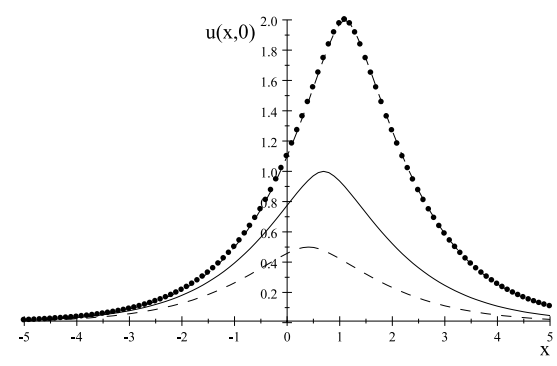

Fig. 2. For $\kappa=1$, the initial values of (a) $w(y, 0)$ and (b) $w(x, 0)$ are plotted with respect to $y$ and $x$, respectively. The specified initial values of (c) $u(y, 0)$ and (d) $u(x, 0)$ are plotted with respect to $y$ and $x$, respectively, at $q_{0}=\frac{1}{3}$ (dash line), $\frac{1}{2}$ (solid line) and $\frac{2}{3}$ (dot dash line).

It is worthy to address here that after performing the above transformation between the coordinates $x$ and $y$, Eq. (2.7) turns out to be exactly identical to the quantum-mechanical Schrödinger equation $-\tilde{\psi}_{y y}+q \tilde{\psi}=k^{2} \tilde{\psi}$. This spectral problem describes the wave function $\tilde{\psi}$ of a quantum particle with the energy $-k^{2}$ in a field with potential $q(y, t)$. Similar to the $\mathrm{KdV}$ equation, construction of the $\mathrm{CH}$ hierarchy with an infinite number of conservation laws becomes theoretically [20] and computationally $[7,28]$ possible. 


\subsection{Spectrum details}

In the following we will extract some physical meanings from the derived scattering data. The first one is the spectrum of $L$. From the discussion stated above, one can see that under the initial condition (2.14), there is only one eigenvalue $\frac{-1}{4} q_{0}^{2}$ for $q_{0} \in(0,1)$. The continuous spectrum is also known to consist of an entire positive real axis. Moreover, provided that $k^{2}=k^{2}\left(q_{0}\right)=\frac{-1}{4} q_{0}^{2}$ we can see that the discrete spectrum $\frac{-1}{4} q_{0}^{2}$ is decreasing w.r.t. $q_{0} \in(0,1)$. This exhibits the relation between the total energy $k^{2}$ and the instantaneous potential $q_{0}$. When the potential $q_{0}$ increases, the total energy $k^{2}$ is decreased and vice versa. In the presence of a larger potential, there is a tendency of encountering a larger potential such that the particle moves slowly. The total energy $k^{2}$ becomes therefore smaller. This interprets the meaning of the scattering data $\mu$ in (2.19). Since $\lambda=\frac{1}{\kappa}\left(\frac{1}{4}+k^{2}\right)$, we can get the spectrum of $H(0)$ which is expressed as $\Sigma(H(0))=\left\{\frac{1}{4 \kappa}\left(1-q_{0}^{2}\right)\right\} \cup$ $\left[\frac{1}{4 \kappa}, \infty\right)$. Moreover, the limiting values of $\frac{1}{4 \kappa}\left(1-q_{0}^{2}\right)$ are obtained respectively from $q_{0} \rightarrow 1$ and 0 as $\lim _{q_{0} \rightarrow 1} \frac{1}{4 \kappa}\left(1-q_{0}^{2}\right)=0$ and $\lim _{q_{0} \rightarrow 0} \frac{1}{4 \kappa}\left(1-q_{0}^{2}\right)=\frac{1}{4 \kappa}$.
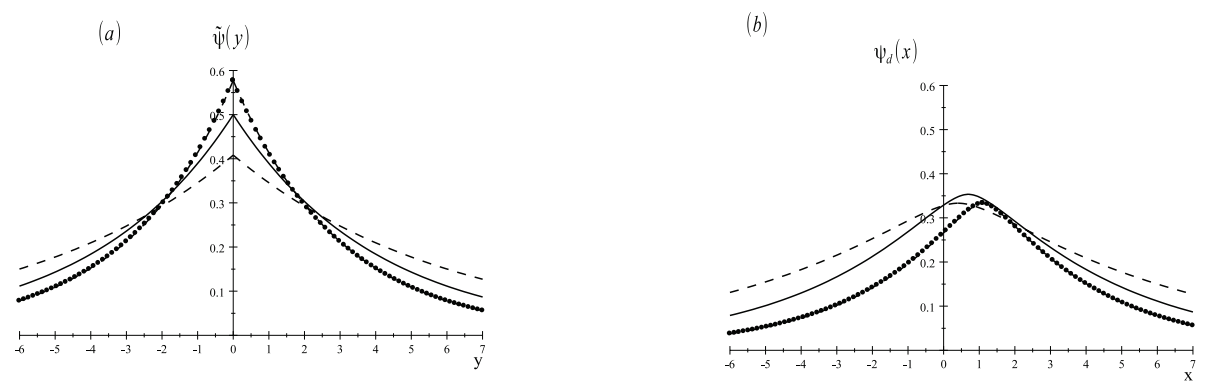

Fig. 3. For $\kappa=1$, the derived eigenfunctions $\tilde{\psi}$ are plotted with respect to $y$ for the case of the discrete spectrum of $L$ in (a) and the eigenfunctions $\psi_{d}$ are plotted with respect to $x$ for the case of the discrete spectrum of $H(0)$ in (b) at $q_{0}=\frac{1}{3}$ (dash line), $\frac{1}{2}$ (solid line) and $\frac{2}{3}$ (dot dash line).

\subsection{Eigenfunction details}

In the following we are aimed to discuss the eigenfunction with respect to the discrete and continuous spectrum, respectively. The derived solution $\tilde{\psi}(y)$ corresponding to the discrete spectrum for the operator $L$ can be expressed as

$$
\tilde{\psi}(y)= \begin{cases}\sqrt{\frac{q_{0}}{2}} e^{-\frac{q_{0}}{2} y}, & y>0, \\ \sqrt{\frac{q_{0}}{2}} e^{\frac{q_{0}}{2} y}, & y<0 .\end{cases}
$$

which is plotted in Figure 3 (a). It is easy to see that the maximum value of $\tilde{\psi}(y)$ takes its presence at $y=0$ since

$$
\max _{y \in \mathbb{R}} \tilde{\psi}(y)=\tilde{\psi}(0)=\sqrt{\frac{q_{0}}{2}} .
$$


Hence as $q_{0}$ increases, the maximum of $\tilde{\psi}(y)$ is increased as well. However, from the result in [2], we have

$$
\gamma^{-2}=\int_{\mathbb{R}} \tilde{\psi}_{+}^{2}\left(y, \frac{q_{0}}{2} i\right) d y=\frac{2}{q_{0}}
$$

where $\tilde{\psi}_{+}(y, k)=e^{i k y}+o(1)$ as $y \rightarrow \infty$. As $q_{0}$ increases, the maximum of $\tilde{\psi}(y)$ is increased accordingly while the $L^{2}$ norm of the eigenfunction $\tilde{\psi}_{+}\left(y, \frac{q_{0}}{2} i\right)$ is decreased. This implies that the mean of the probability distribution of the wavefunction becomes smaller. The meaning of the scattering data $\gamma$ in Section 2.19 becomes more clear to us.

Now we intend to interpret the meaning of the total energy $k^{2}$. More precisely, we are aimed to make a connection between the discrete spectrum $k_{1}^{2}=-\mu^{2}$ with $\mu$ defined in (2.19) and the corresponding eigenfunction $\tilde{\psi}_{1}$. From (2.18) we know

$$
-\tilde{\psi}_{1, y y}+q(y, 0) \tilde{\psi}_{1}=k_{1}^{2} \tilde{\psi}_{1} .
$$

Multiplying both sides of (3.1) by $\tilde{\psi}_{1}$ and integrating the resulting equation from $-\infty$ to $\infty$ by virtue of the integration by parts, we can obtain

$$
\int_{\mathbb{R}}\left|\tilde{\psi}_{1, y}(y)\right|^{2} d y+\int_{\mathbb{R}} q(y, 0)\left|\tilde{\psi}_{1}(y)\right|^{2} d y=k_{1}^{2} \int_{\mathbb{R}} \tilde{\psi}_{1}^{2}(y) d y .
$$

In the above derivation we make use of the property that $\tilde{\psi}_{1}$ will be decayed to zero at $\pm \infty$. Application of the normalization condition in (2.10) yields

$$
k_{1}^{2}=\int_{\mathbb{R}}\left|\tilde{\psi}_{1, y}(y)\right|^{2} d y-q_{0}\left|\tilde{\psi}_{1}(0)\right|^{2} .
$$

One can regard $-q_{0}\left|\tilde{\psi}_{1}(0)\right|^{2}$ as the potential energy and $\int_{\mathbb{R}}\left|\tilde{\psi}_{1, y}(y)\right|^{2} d y$ as the kinetic energy.

For the eigenfunction $\psi_{d}(x)$ corresponding to the discrete spectrum of the operator $H(0)$, the result is quite different. By (2.5) and (2.6), $\psi_{d}(x)$ can be expressed as

$$
\psi_{d}(x)= \begin{cases}\sqrt{\frac{q_{0}}{2}}\left(\frac{1}{1-\frac{q_{0}}{1-q_{0}} e^{-x}}\right)^{-\frac{1}{2}} \frac{1}{\left(e^{x}-\frac{q_{0}}{1-q_{0}}\right)^{\frac{q_{0}}{2}}}, & \text { if } x>\ln \left(1+\frac{q_{0}}{1-q_{0}}\right), \\ \sqrt{\frac{q_{0}}{2}}\left(\frac{\left(1+\frac{q_{0}}{1-q_{0}}\right)^{2}}{\left(1+\frac{q_{0}}{1-q_{0}}\right)^{2}-\frac{q_{0}}{1-q_{0}} e^{x}}\right)^{-\frac{1}{2}} \frac{1}{\left(\left(1+\frac{q_{0}}{1-q_{0}}\right)^{2} e^{-x}-\frac{q_{0}}{1-q_{0}}\right)^{\frac{q_{0}}{2}}}, & \text { if } x \leq \ln \left(1+\frac{q_{0}}{1-q_{0}}\right) .\end{cases}
$$

The maximum of $\psi_{d}(x)$, which is $\sqrt{\frac{q_{0}\left(1-q_{0}\right)}{2}}$ is present at $x=\ln \left(1+\frac{q_{0}}{1-q_{0}}\right)$. Hence when $q_{0}=\frac{1}{2}$, $\psi_{d}(x)$ has the largest amplitude shown in Figure 3 (b).

For a continuous spectrum, we demand the satisfaction of the following eigenfunction $\hat{\psi}=$ $\hat{\psi}(y, k)$ from the derivation of scattering data in [16]:

$$
\hat{\psi}(y, k)= \begin{cases}e^{-i k y}+\tilde{R}(k) e^{i k y}, & y>0, \\ \tilde{T}(k) e^{-i k y} & y<0 .\end{cases}
$$


Then the eigenfunction $\hat{\psi}(y, k)$ corresponding to the continuous spectrum of the operator $L$ can be expressed as

$$
\begin{gathered}
\operatorname{Re} \hat{\psi}(y, k)=\left\{\begin{array}{cc}
\frac{4 k^{2}}{q_{0}^{2}+4 k^{2}} \cos k y-\frac{2 q_{0} k}{q_{0}^{2}+4 k^{2}} \sin k y, & y \geq 0, \\
\frac{4 k^{2}}{q_{0}^{2}+4 k^{2}} \cos k y+\frac{2 q_{0} k}{q_{0}^{2}+4 k^{2}} \sin k y, & y<0,
\end{array}\right. \\
\operatorname{Im} \hat{\psi}(y, k)= \begin{cases}\frac{2 q_{0} k}{q_{0}^{2}+4 k^{2}} \cos k y-\left(\frac{2 q_{0}^{2}+4 k^{2}}{q_{0}^{2}+4 k^{2}}\right) \sin k y, & y \geq 0, \\
\frac{2 q_{0} k}{q_{0}^{2}+4 k^{2}} \cos k y-\frac{4 k^{2}}{q_{0}^{2}+4 k^{2}} \sin k y, & y<0 .\end{cases}
\end{gathered}
$$

In Figure 4 (a) and 4 (b), the graphs of $\operatorname{Re} \hat{\psi}(y, k)$ and $\operatorname{Im} \hat{\psi}(y, k)$ are plotted respectively with respect to $k$ and $y$ at $q_{0}=\frac{1}{2}$. Moreover, in Figure 4 (c), the graphs of $\operatorname{Re} \hat{\psi}(y, k)$ are plotted at different wavenumbers $k=\frac{3}{8}, \frac{5}{8}, \frac{3}{2}$ and $q_{0}=\frac{1}{2}$. In Figure 4 (d), the graphs of $\operatorname{Re} \hat{\psi}(y, k)$ are plotted at the wavenumber $k=1$ for $q_{0}=\frac{1}{3}, \frac{1}{2}, \frac{2}{3}$. In particular, from Figure 4 (d) we can see that for different values of $q_{0}$ their profiles of $\operatorname{Re} \hat{\psi}(y, k)$ do not change obviously. It can be seen in Figure 4 (a) that $\operatorname{Re} \hat{\psi}(y, k)$ is an even function with respect to $k$. Similar cases for $\operatorname{Im} \hat{\psi}(y, k)$ are also plotted in Figure 4 (e), Figure 4 (f) and Figure 4 (g), respectively. It can be seen in Figure 4 (b) that $\operatorname{Im} \hat{\psi}(y, k)$ is an odd function with respect to $k$.

By the Liouville transformation, the eigenfunction $\psi_{c}(x, k)$ corresponding to the continuous spectrum for the operator $H(0)$ can be expressed as

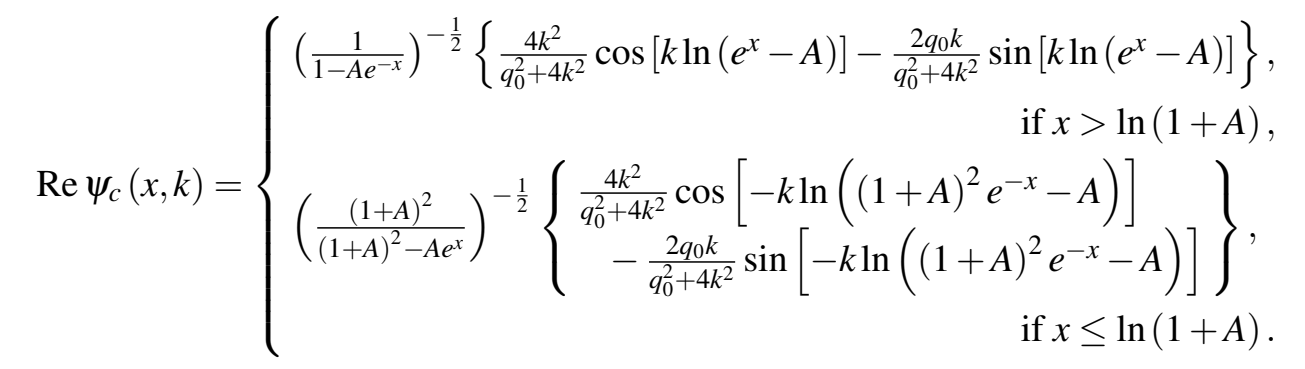

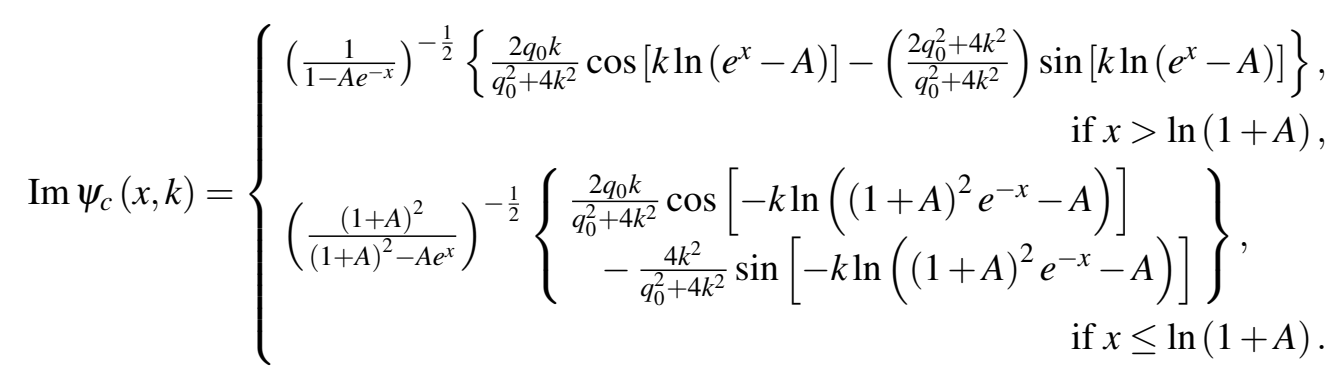

In Figure 5 (a) and Figure 5 (b), the graphs of $\operatorname{Re} \psi_{c}(x, k)$ and $\operatorname{Im} \psi_{c}(x, k)$ are plotted respectively with respect to the wavenumber $k$ and $x$. Moreover, in Figure 5 (c), the graphs of $\operatorname{Re} \psi_{c}(x, k)$ are plotted at different wavenumbers $k=\frac{3}{8}, \frac{5}{8}, \frac{3}{2}$ at $q_{0}=\frac{1}{2}$. In Figure $5(\mathrm{~d})$, the graphs of $\operatorname{Re} \psi_{c}(x, k)$ are plotted at the wavenumber $k=1$ for $q_{0}=\frac{1}{3}, \frac{1}{2}, \frac{2}{3}$. It can be seen in Figure 5 (a) that $\operatorname{Re} \psi_{c}(x, k)$ is an even function with respect to $k$. For $\operatorname{Im} \psi_{c}(x, k)$ they are also plotted in Figure 5 (e), Figure 5 (f) and Figure 5 (g), respectively. It can be seen from Figure 5 (b) that $\operatorname{Im} \psi_{c}(x, k)$ is an odd function with respect to $k$. 

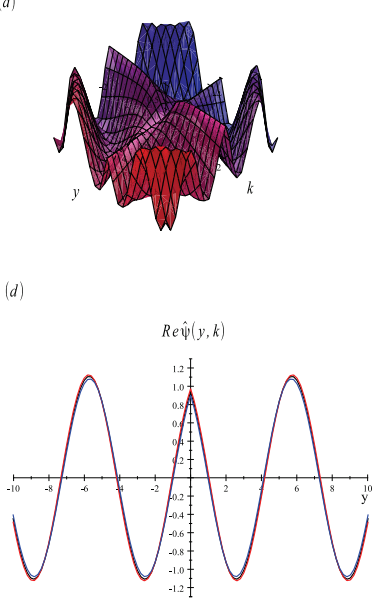

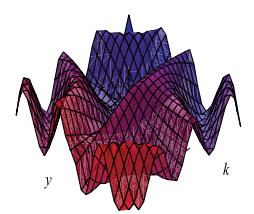

$(e)$
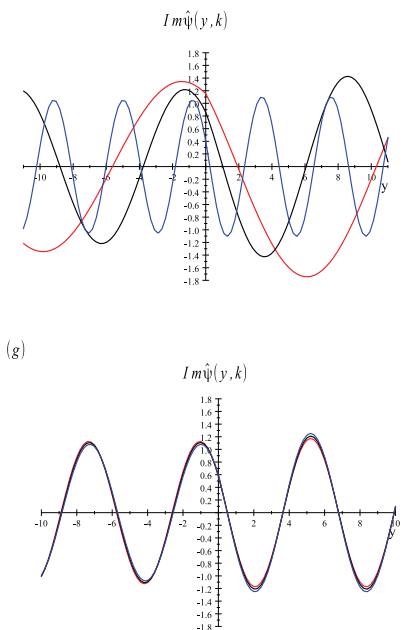

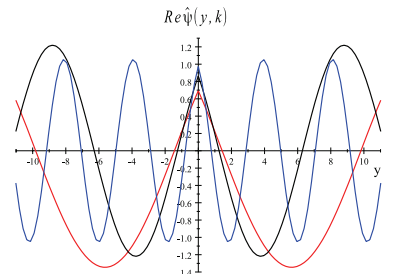

$(f)$

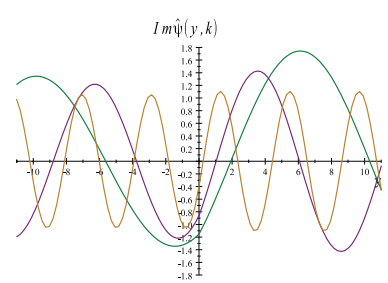

Fig. 4. For $\kappa=1$ : (a) $\operatorname{Re} \hat{\psi}(y, k)$; (b) $\operatorname{Im} \hat{\psi}(y, k)$ at $q_{0}=\frac{1}{2}$; (c) $\operatorname{Re} \hat{\psi}(y, k)$ for $k=\frac{3}{8}$ (red line), $\frac{5}{8}$ (black line), $\frac{3}{2}$ (blue line) at $q_{0}=\frac{1}{2}$; (d) $\operatorname{Re} \hat{\psi}(y, k)$ for $k=1$ at $q_{0}=\frac{1}{3}$ (red line), $\frac{1}{2}$ (black line), $\frac{2}{3}$ (blue line); (e) $\operatorname{Im} \hat{\psi}(y, k)$ for $k=\frac{3}{8}$ (red line), $\frac{5}{8}$ (black line), $\frac{3}{2}$ (blue line) at $q_{0}=\frac{1}{2}$; (f) $\operatorname{Im} \hat{\psi}(y, k)$ for $k=-\frac{3}{8}$ (green line), $-\frac{5}{8}$ (purple line), $-\frac{3}{2}$ (yellow line), at $q_{0}=\frac{1}{2} ;(\mathrm{g}) \operatorname{Im} \hat{\psi}(y, k)$ for $k=1$ at $q_{0}=\frac{1}{3}$ (red line), $\frac{1}{2}$ (black line), $\frac{2}{3}$ (blue line).

\subsection{Transmission and reflection coefficients}

In the following the transmission and reflection coefficients $T(k)$ and $R(k)$ are discussed separately. Since (2.13) holds, $\tilde{R}(k)$ becomes the same as $R(k)$ and only the data $R(k)$ will be considered. From (2.19), the graphs of $\operatorname{Re}(R(k)), \operatorname{Im}(R(k))$ and $|R(k)|^{2}$ are shown in Figure 6 (a), 6 (b) and 6 (c), respectively.

For the transmission coefficient $\tilde{T}(k)$, from (2.19) and (3.2), we have $\tilde{T}(k)=1+\tilde{R}(k)$ or

$$
\tilde{T}(k)=\frac{2 i k}{q_{0}+2 i k} \text {. }
$$

The graphs of $\operatorname{Re}(\tilde{T}(k)), \operatorname{Im}(\tilde{T}(k))$ and $|\tilde{T}(k)|^{2}$ are plotted with respect to the wavenumber $k$ in Figure 6 (d), 6 (e) and 6 (f), respectively. From (2.13) we can have $T(k)=\tilde{T}(k) e^{i k H_{-1}(w)}$. Hence, the transmission coefficient is expressed as $T(k)=\frac{2 i k}{q_{0}+2 i k} e^{i k \ln (1+A)^{2}}$, or

$$
\begin{aligned}
& \operatorname{Re}(T(k))=\frac{4 k^{2}}{q_{0}^{2}+4 k^{2}} \cos \left(\left(\ln \left(1+\frac{q_{0}}{1-q_{0}}\right)^{2}\right) k\right)-\frac{2 q_{0} k}{q_{0}^{2}+4 k^{2}} \sin \left(\left(\ln \left(1+\frac{q_{0}}{1-q_{0}}\right)^{2}\right) k\right), \\
& \operatorname{Im}(T(k))=\frac{2 q_{0} k}{q_{0}^{2}+4 k^{2}} \cos \left(\left(\ln \left(1+\frac{q_{0}}{1-q_{0}}\right)^{2}\right) k\right)+\frac{4 k^{2}}{q_{0}^{2}+4 k^{2}} \sin \left(\left(\ln \left(1+\frac{q_{0}}{1-q_{0}}\right)^{2}\right) k\right) .
\end{aligned}
$$


$(a)$

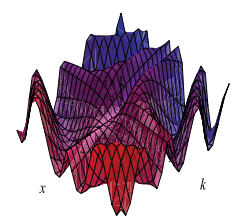

$(d)$

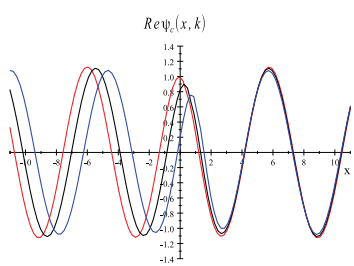

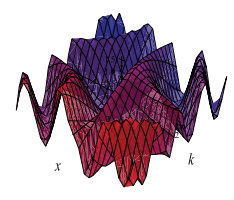
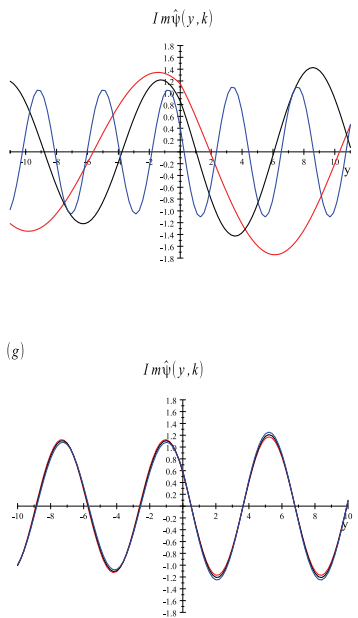

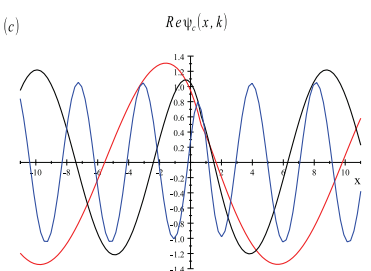

$(f)$

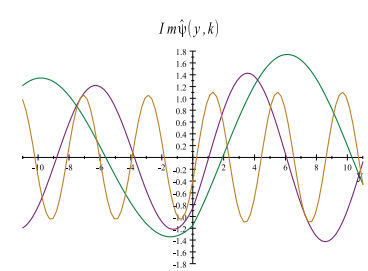

Fig. 5. For $\kappa=1$ : (a) $\operatorname{Re} \psi_{c}(x, k)$ and (b) $\operatorname{Im} \psi_{c}(x, k)$ at $q_{0}=\frac{1}{2}$; (c) $\operatorname{Re} \psi_{c}(x, k)$ for $k=\frac{3}{8}$ (red line), $\frac{5}{8}$ (black line), $\frac{3}{2}$ (blue line) at $q_{0}=\frac{1}{2}$; (d) $\operatorname{Re} \psi_{c}(x, k)$ are plotted with respect to $x$ with the wavenumber $k=1$ at $q_{0}=\frac{1}{3}$ (red line), $\frac{1}{2}$ (black line), $\frac{2}{3}$ (blue line); (e) Im $\psi_{c}(x, k)$ for the wavenumbers $k=\frac{3}{8}$ (red line), $\frac{5}{8}$ (black line), $\frac{3}{2}$ (blue line) at $q_{0}=\frac{1}{2}$; (f) $\operatorname{Im} \psi_{c}(x, k)$ for the wavenumbers $k=-\frac{3}{8}$ (green line), $-\frac{5}{8}$ (purple line), $-\frac{3}{2}$ (yellow line) at $q_{0}=\frac{1}{2} ;(\mathrm{g}) \operatorname{Im} \psi_{c}(x, k)$ for $k=1$ at $q_{0}=\frac{1}{3}$ (red line), $\frac{1}{2}$ (black line), $\frac{2}{3}$ (blue line).

The graphs of the above two expressions are shown in Figure $6(\mathrm{~g})$ and $6(\mathrm{~h})$, respectively. Finally, in Figure 6 (i) we plot $|R(k)|^{2}$ and $|T(k)|^{2}$ to reveal how the reflection and transmission coefficients are varied with the wavenumber $k$ and the specified potential $q_{0}$. From the graphs of the transmission and reflection coefficients, the dependence of these coefficients on the strength of potential $q_{0}$ and the wavenumber $k$ is summarized as follows:

1. From Figure 6 (c) and 6 (f) for the graphs of $|R(k)|^{2}$ and $|T(k)|^{2}$, one knows that when $q_{0}$ becomes larger, the magnitude of $|R(k)|^{2}$ will be larger and the magnitude of $|T(k)|^{2}$ becomes, on the contrary, smaller for all $k \in \mathbb{R}$. This result can be interpreted as follows: When the instantaneous potential $q_{0}$ becomes larger, the right-running incident wave which behaves like $e^{-i k y}$ as $y \rightarrow \infty$ does not have enough kinetic energy to transmit itself to the left (i.e., $|T(k)|^{2}$ becomes smaller) and a larger portion of this wave will be reflected (i.e., $|R(k)|^{2}$ becomes larger).

2. For a fixed value of $q_{0}$, the reflection coefficient $|R(k)|^{2}$ will be concentrated in the neighborhood of $k=0$. That is, $|R(k)|^{2}$ turns out to be zero locally as $k \rightarrow \pm \infty$. On the other hand, the transmission coefficient $|T(k)|^{2}$ will decay to zero near $k=0$. This finding can be interpreted as follows: When the total energy $|k|^{2}$ becomes larger, the right-running incident wave will have sufficient kinetic energy to transmit. In other words, only a small percentage of the right-running incident wave will be reflected. 
Based on the above two observations, one can see that the prescribed instantaneous potential $q_{0}$ and the derived total energy $k^{2}$ are the two essential factors of guiding the transmission behavior of the wave function $\tilde{\psi}$ in (2.18).
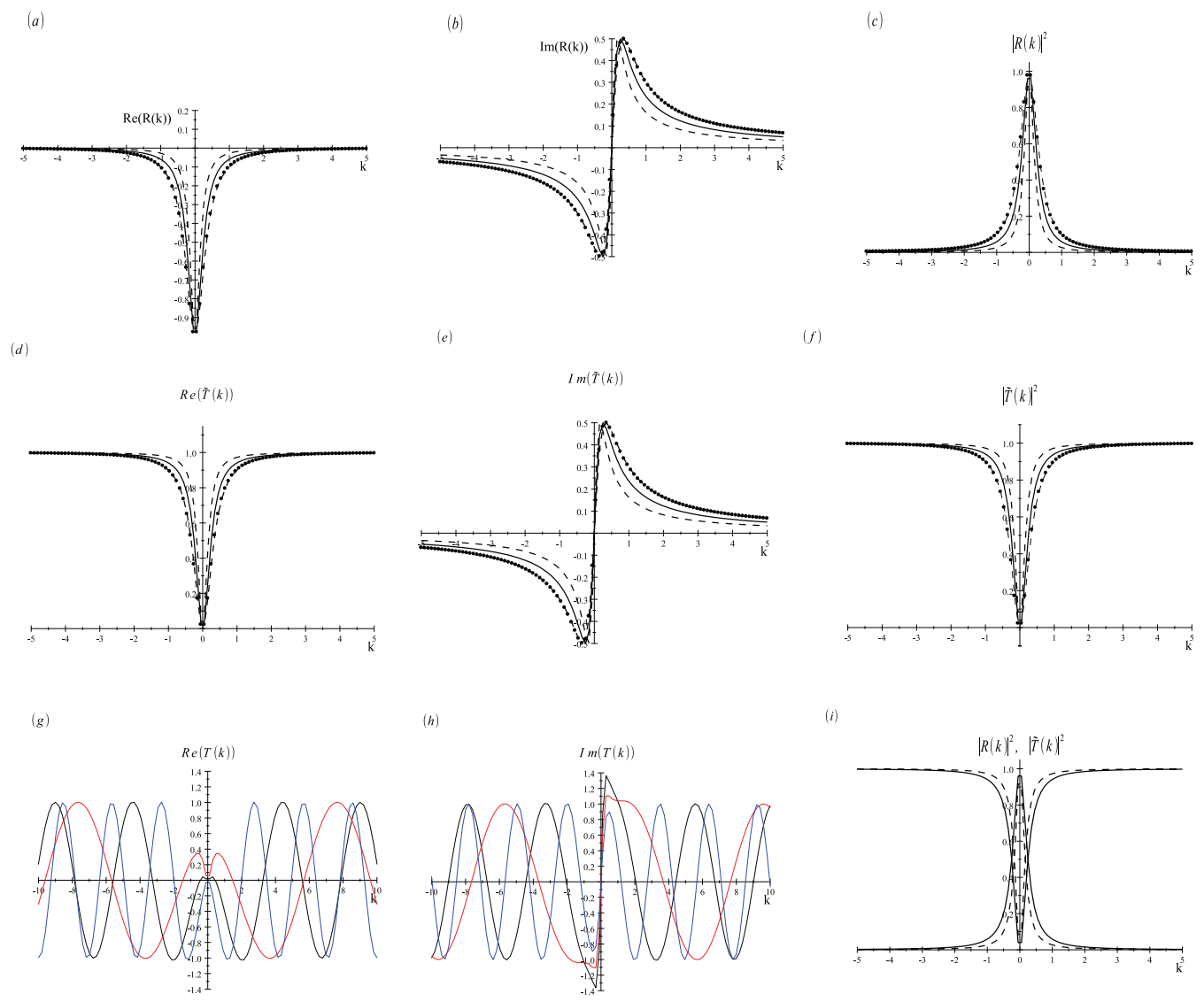

Fig. 6. For $\kappa=1$ : (a) Re $\mathrm{R}(k)$ and (b) $\operatorname{Im} \mathrm{R}(k)$; (c) The magnitudes $|R(k)|^{2}$ for $q_{0}=\frac{1}{3}$ (dash line), $\frac{1}{2}$ (solid line), $\frac{2}{3}$ (dot dash line); (d) $\operatorname{Re} \tilde{T}(k)$ and (e) $\operatorname{Im} \tilde{T}(k)$; (f) The magnitudes of $|\tilde{T}(k)|^{2}$ at $q_{0}=\frac{1}{3}$ (dash line), $\frac{1}{2}$ (solid line), $\frac{2}{3}$ (dot dash line); $(\mathrm{g}) \operatorname{Re} T(k)$ and (h) $\operatorname{Im} T(k)$ for $q_{0}=\frac{1}{3}$ (red line), $\frac{1}{2}$ (black line), $\frac{2}{3}$ (blue line); (i) The magnitudes of $|R(k)|^{2}$ and $|\tilde{T}(k)|^{2}$ at $q_{0}=\frac{1}{3}$ (dash line) and $\frac{1}{2}$ (solid line).

\section{Conclusion}

Our goal of getting some missing details of wave propagation by FDTD simulation motivates the current spectral analysis of the Camassa-Holm equation. Our aim is to extract the scattering wave nature of initial condition using the Lax pair equations of the eigenvalue type. Through the Liouville transformation, we get the linear Schrödinger equation cast in the transformed coordinate. As a result, the continuous and discrete spectra can be both rigorously analyzed within the context of the direct scattering spectral analysis, thereby enabling us to get their respective scattering solution and the bound state. The major conclusions drawn from the current study are summarized as follows: 
The potential $q(y, 0)$ in $(2.18)$ has a form of Delta function. Although the result of the scattering data can be found as in [16], there are still many interesting aspects that have not been discussed before:

1. It is interesting to consider the initial condition which takes the Delta function form in $y$ coordinate. The answer can be easily found in [16] for instance. However, it is not a straightforward task for the analysis of $\mathrm{CH}$ equation. We need to solve $w$ in (2.8) at $t=0$ with $q(y, 0)$ being known to be the Delta function. Then we need to find the relation $y=y(x)$ between the $x$ domain and the $y$ domain in (2.6). Finally, we need to solve $u(x, 0)$ from the equation defining the momentum in (2.3) with $w(x, 0)=\left.w(y, 0)\right|_{y=y(x)}$. The exact solutions of the above three ordinary differential equations can be found. This is our motivation to derive the initial condition in (2.14).

2. While the scattering data found in (2.19) can be seen in [16], its detailed wave physics is discussed for the first time in Section 4. Our study is aimed to know the functional dependence of the scattering data on the strength of the instantaneous potential $-q_{0} \delta(y)$ in (2.18). Through the analytically derived results plotted in the figures of this article, we are led to know how the strength $q_{0}$ can affect the wavefunctions and the scattering data.

In a forthcoming paper, we will make a comparison of the solution computed directly from the initial value problem (1.1) and (2.14) using the finite difference method and the long-time asymptotics from [2] and [1]. Based on the work carried out in this paper, we have derived a new initial condition which can indeed help us to learn the true properties of the scattering data. This viewpoint may be new and we believe this work could be interesting to some readers.

\section{Acknowledgments}

The authors would like to thank Professor Chang-Shou Lin for his fruitful discussion in the course of conducting this study. Financial support under 101-2745-M-002-004-ASP is also acknowledged.

\section{References}

[1] A. Boutet de Monvel, A. Its, and D. Shepelsky, Painlevé-type asymptotics for the Camassa-Holm equation, SIAM J. Math. Anal. 42 (2010) 1854-1873.

[2] A. Boutet de Monvel, A. Kostenko, D. Shepelsky, and G. Teschl, Long- time asymptotics for the Camassa-Holm equation, SIAM J. Math. Anal. 41 (2009) 1559-1588.

[3] R. Camassa, P. H. Chiu, L. Lee, and T. W. H. Sheu, Viscous and inviscid regularizations in a class of evolutionary partial differential equations, J. Comput. Phys. 229 (2010) 6676-6687.

[4] R. Camassa, P. H. Chiu, L. Lee, and T. W. H. Sheu, A particle method and numerical study of a quasilinear partial differential equation, Commun. Pure Appl. Anal. 10 (2011) 1503-1515.

[5] R. Camassa and D. D. Holm, An integrable shallow water equation with peaked solitons, Phys. Rev. Lett. 71 (1993) 1661-1664.

[6] R. Camassa, D. D. Holm, and J. M. Hyman, A new integrable shallow water equation, Advances in Applied Mechanics 31 (1994), pp. 1-33.

[7] P. H. Chiu, L. Lee, and T. W. H. Sheu, A sixth-order dual preserving algorithm for the Camassa-Holm equation, J. Comput. Appl. Math. 233 (2010) 2767-2778.

[8] A. Constantin, Existence of permanent and breaking waves for a shallow water equation: a geometric approach, Ann. Inst. Fourier (Grenoble), 50 (2000) 321-362.

[9] A. Constantin, On the scattering problem for the Camassa-Holm equation, R. Soc. Lond. Proc. Ser. A Math. Phys. Eng. Sci. 457 (2001) 953-970.

[10] A. Constantin and J. Escher, Global existence and blow-up for a shallow water equation, Ann. Scuola Norm. Sup. Pisa Cl. Sci. (4) 26 (1998) 303-328. 
[11] A. Constantin and J. Escher, Global weak solutions for a shallow water equation, Indiana Univ. Math. J. 47 (1998) 1527-1545.

[12] A. Constantin and J. Escher, Wave breaking for nonlinear nonlocal shallow water equations. Acta Math. 181 (1998) 229-243.

[13] A. Constantin and D. Lannes, The hydrodynamical relevance of the Camassa-Holm and DegasperisProcesi equations. Arch. Rantion. Mech. Anal. 192 (2009) 165-186.

[14] A. Constantin and W. A. Strauss, Stability of peakons. Comm. Pure Appl. Math. 53 (2000) 603-610.

[15] A. Constantin and W. A. Strauss, Stability of the Camassa-Holm solitons. J. Nonlinear. Sci. 12 (2002) 415-422.

[16] P. G. Drazin and R. S. Johnson, Solitons: an introduction, Cambridge Texts in Applied Mathematics. (Cambridge University Press, Cambridge, 1989).

[17] L. D. Faddeev, On the relation between the S-matrix and potential for the one-dimensional Schrödinger operator, Dokl. Akad. Nauk SSSR 121 (1958) 63-66.

[18] B. Fuchssteiner and A. Fokas, Symplectic structures, their Bäcklund transformation and hereditary symmetries, Phys. D 4 (1981/82) 47-66.

[19] C. S. Gardner, J. M. Green, M. D. Kruskal and R. M. Miura, Korteweg-de Vries equation and generalizations. VI. Methods for exact solution, Comm. Pure Appl. Math. 27 (1974) 97-133.

[20] F. Gesztesy and H. Holden, Soliton Equations and their Algebro-Geometric Solutions Vol. I, Cambridge Studies in Advanced Mathematics 79 (Cambridge University Press, Cambridge, 2003. $(1+1)$ dimensional continuous models).

[21] D. Ionescu-Krus, Variational derivation of the Camassa-Holm shallow water equation, J. Nonlinear Math. Phys. 14 (2007) 311-320.

[22] R. S. Johnson, Camassa-Holm, Korteweg-de Vries and related models for water waves, J. Fluid Mech. 455 (2002) 63-82.

[23] R. S. Johnson, On solutions of the Camassa-Holm equation, R. Soc. Lond. Proc. Ser. A Math. Phys. Eng. Sci. 459 (2003) 1687-1708.

[24] J. Lenells, The scattering approach for the Camassa-Holm equation, J. Nonlinear Math. Phys. 9 (2002) 389-393.

[25] Y. Li and J. E. Zhang, The multiple-soliton solution of the Camassa-Holm equation, R. Soc. Lond. Proc. Ser. A Math. Phys. Eng. Sci. 460 (2004) 2617-2627.

[26] V. A. Marchenko, Sturm-Liouville Operators and Applications, Operator Theory: Advances and Applications 22 (Birkhäuser Verlag, Basel, 1986, Translated from the Russian by A. Iacob).

[27] D. McMahon, Quantum Mechanics Demystified (McGraw-Hill Professional Publishing, New York, USA 2006).

[28] T. W. H. Sheu, P. H. Chiu, and C. H. Yu, On the development of a high-order compact scheme for exhibiting the switching and dissipative solution natures in the Camassa-Holm equation, J. Comput. Phys. 230 (2011) 5399-5416.

[29] G. B. Whitham, Linear and nonlinear waves ( Wiley-Interscience [John Wiley \& Sons], New YorkLondon-Sydney, 1974. Pure and Applied Mathematics). 